\title{
3HSP J095507.9+355101: A flaring extreme blazar coincident in space and time with IceCube-200107A
}

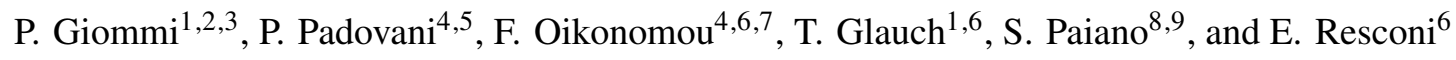 \\ ${ }^{1}$ Institute for Advanced Study, Technische Universität München, Lichtenbergstrasse 2a, 85748 Garching bei München, Germany \\ e-mail: giommipaolo@gmail.com \\ 2 Associated to Agenzia Spaziale Italiana, ASI, Via del Politecnico s.n.c., 00133 Roma, Italy \\ 3 ICRANet, Piazzale della Repubblica 10, 65122 Pescara, Italy \\ 4 European Southern Observatory, Karl-Schwarzschild-Str. 2, 85748 Garching bei München, Germany \\ 5 Associated to INAF - Osservatorio Astronomico di Roma, Via Frascati 33, 00040 Monteporzio Catone, Italy \\ 6 Technische Universität München, Physik-Department, James-Frank-Str. 1, 85748 Garching bei München, Germany \\ 7 Institutt for fysikk, NTNU, Trondheim, Norway \\ 8 INAF - Osservatorio Astronomico di Roma, Via Frascati 33, 00040 Monteporzio Catone, Italy \\ 9 INAF - IASF Milano, Via Corti 12, 20133 Milano, Italy
}

Received 15 May 2020 / Accepted 5 July 2020

\begin{abstract}
The uncertainty region of the highly energetic neutrino IceCube200107A includes 3HSP J095507.9+355101 $(z=0.557)$, an extreme blazar, which was detected in a high, very hard, and variable X-ray state shortly after the neutrino arrival. Following a detailed multiwavelength investigation, we confirm that the source is a genuine BL Lac. This new detection differs from TXS 0506+056, which is thus far the first source associated with IceCube neutrinos, and is considered a "masquerading" BL Lac. As in the case of TXS 0506+056, 3HSP J095507.9+355101 is also way off the so-called blazar sequence. We consider 3HSP J095507.9+355101 a possible counterpart to the IceCube neutrino. Finally, we discuss some theoretical implications in terms of neutrino production.
\end{abstract}

Key words. neutrinos - radiation mechanisms: non-thermal - galaxies: active - gamma rays: galaxies - BL Lacertae objects: general

\section{Introduction}

The IceCube Neutrino Observatory at the South Pole ${ }^{1}$ has detected tens of high-energy neutrinos of likely astrophysical origin (e.g. IceCube Collaboration 2017a; Schneider 2020; Stettner 2020, and references therein). So far, only one astronomical object has been significantly associated (in space and time) with some of these neutrinos, that is the bright blazar TXS 0506+056 at $z=0.3365$ (IceCube Collaboration 2018a,b; Padovani et al. 2018; Paiano et al. 2018). It is clear, however, that blazars cannot be responsible for the whole IceCube signal (see IceCube Collaboration 2017b; Aartsen et al. 2017). The case for some blazars being neutrino sources, however, is mounting. Several studies have reported hints of a correlation between blazars and the arrival direction of astrophysical neutrinos (e.g. Padovani \& Resconi 2014; Padovani et al. 2016; Lucarelli et al. 2019 and references therein) and possibly of ultra high-energy cosmic rays (Resconi et al. 2017). Moreover, very recently some of the authors of this Letter (Giommi et al. 2020a) have extended the detailed dissection of the region around the IceCube-170922A event related to TXS 0506+056 carried out by Padovani et al. (2018) to all the 70 public IceCube high-energy neutrinos that are well reconstructed (so-called tracks) and off the Galactic plane. This resulted in a $3.23 \sigma$ (post-trial) excess of

\footnotetext{
1 http://icecube.wisc.edu
}

IBLs $^{2}$ and HBLs with a best fit of $15 \pm 4$ signal sources, while no excess was found for LBLs. Given that TXS 0506+056 is also a blazar of the IBL/HBL type (Padovani et al. 2019) this result, together with previous findings, consistently points to growing evidence for a connection between some IceCube neutrinos and IBL and HBL blazars. We report on 3HSP J095507.9+355101, an HBL within the error region of the IceCube track IceCube200107A (see Fig. 1), which was found to exhibit an X-ray flare the day after the neutrino arrival. This source belongs to the third high-synchrotron peaked (3HSP) catalogue (Chang et al. 2019), which includes blazars with $v_{\text {peak }}^{S}>10^{15} \mathrm{~Hz}$. Actually, with a catalogued synchrotron peak frequency of $\sim 5 \times 10^{17} \mathrm{~Hz}$, and a significantly higher value during the flare (Sect. 2.2), this source belongs to the rare class of extreme blazars (e.g. Biteau et al. 2020, and references therein). We also comment on the nature of the source and the theoretical implications in terms of neutrino production. We use a $\Lambda$ CDM cosmology with $H_{0}=70 \mathrm{~km} \mathrm{~s}^{-1} \mathrm{Mpc}^{-1}, \Omega_{\mathrm{m}, 0}=0.3$, and $\Omega_{\Lambda, 0}=0.7$.

\footnotetext{
2 Blazars are divided based on the rest-frame frequency of the lowenergy (synchrotron) hump ( $\left.v_{\text {peak }}^{S}\right)$ into LBL/LSP sources $\left(v_{\text {peak }}^{S}<\right.$ $\left.10^{14} \mathrm{~Hz} \quad[<0.41 \mathrm{eV}]\right)$, intermediate- $\left(10^{14} \mathrm{~Hz}<v_{\text {peak }}^{S}<10^{15} \mathrm{~Hz}\right.$ $[0.41 \mathrm{eV}-4.1 \mathrm{eV}])$, and high-energy $\left(v_{\text {peak }}^{S}>10^{15} \mathrm{~Hz}[>4.1 \mathrm{eV}]\right)$ peaked (IBL/ISP and HBL/HSP) sources, respectively (Padovani \& Giommi 1995; Abdo et al. 2010).
} 


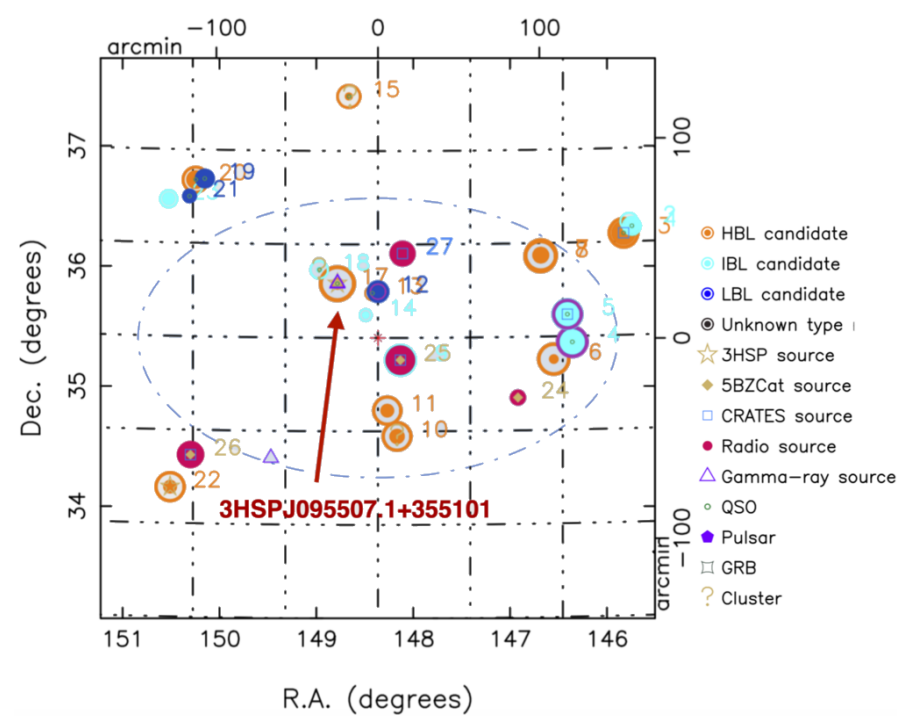

Fig. 1. Known and candidate blazars (radio/X-ray matching sources) around the $90 \%$ containment region of IceCube200107A, approximated by the elliptical area delimited by the dot-dashed curve.

\section{Multi-messenger data}

\subsection{IceCube data}

On January 7, 2020 the IceCube Collaboration reported the detection of a high-energy neutrino candidate (HESE; Stein 2020) of possible astrophysical origin. While the event was not selected by the standard real-time detection procedure, it was identified as a starting track by a newly developed deep neuralnetwork event classifier (Kronmueller \& Glauch 2020). After applying off-line reconstructions, the arrival direction is given, as right ascension, $148.18_{-1.83}^{+2.20} \mathrm{deg}$ and declination $35.46_{-1.22}^{+1.10} \mathrm{deg}$ at $90 \%$ C.L. As this was an unscheduled report, IceCube does not provide any energy information. Assuming an $E^{-2}$ spectrum and the effective area for HESE starting tracks (IceCube Collaboration 2014), at this declination $90 \%$ of neutrinos have energy $0.33_{-0.27}^{+2.23} \mathrm{PeV}^{3}$. Further evidence that the event is astrophysical comes from the direction. Because the event comes from the Northern Hemisphere, an atmospheric muon origin can be excluded. Also the fraction of the conventional atmospheric muon neutrino background is suppressed compared to the horizon. In a follow-up GCN report (Pizzuto 2020), IceCube announced the detection of two additional neutrino candidates in spatial coincidence with the $90 \%$ containment region of IceCube-200107A in a time range of two days around the alert consistent with atmospheric background at a $4 \%$ level. We note that the error region of IceCube-200107A is also fully inside the $16.5^{\circ}$ median angular error circle of a HESE shower detected by IceCube in 2011 (HES9), and reported in IceCube Collaboration (2014). In fact, 3HSP J095507.9+355101 is located only $0.62^{\circ}$ and $2.73^{\circ}$ away from the best-fit position of IceCube-200107A and HES9, respectively.

We estimate the flux required to detect, on average, one muon neutrino with IceCube at a specified time interval, $\Delta T$, by assuming the neutrino event, IceCube-200107A, to be a signal event. The number of signal-only, muon (and antimuon) neutrinos detected during $\Delta T$ at declination $\delta$ is given by $N_{v_{\mu}}=$ $\int_{E_{\nu_{\mu}, \min }}^{E_{\nu_{\mu}, \max }} \mathrm{d} E_{\gamma_{\mu}} A_{\mathrm{eff}}\left(E_{v_{\mu}}, \delta\right) \phi_{E_{v_{\mu}}} \Delta T$, where $E_{\nu, \text { min }}$ and $E_{\nu, \text { max }}$, are the

\footnotetext{
3 For an assumed $E^{-1} / E^{-2.7}$ neutrino spectrum, $90 \%$ of neutrinos have
} energy $1.40_{-1.22}^{+5.75} \mathrm{PeV} / 0.16_{-0.12}^{+0.83} \mathrm{PeV}$ respectively.
$90 \%$ C.L. lower and upper limits on the energy of the neutrino, respectively, $A_{\text {eff }}$ is the effective area, and $\phi_{E_{v_{\mu}}}$ the muon neutrino differential energy flux. We assume a source emitting an $E^{-2}$ neutrino spectrum between $65 \mathrm{TeV}$ and $2.6 \mathrm{PeV}$, the energy range in which we expect $90 \%$ of neutrinos detected from the direction of IC-200107A in the HESE channel. Since the neutrino emission duration is unknown we calculate the neutrino flux needed to produce one neutrino in IceCube from the direction of IceCube-200107A for $\Delta T=30 \mathrm{~d} / 250 \mathrm{~d} / 10 \mathrm{yr}$, corresponding to the lower limit on the duration of the UV/soft X-ray flare (Sect. 2.2), the Fermi integration time (Sect. 2.4), and the duration of the IceCube operation, respectively. Using the effective area of Blaufuss et al. (2020), we obtain an integrated all-flavour neutrino energy flux of $3 \times 10^{-9} / 4 \times 10^{-10} / 3 \times 10^{-11} \mathrm{erg} \mathrm{cm}^{-2} \mathrm{~s}^{-1}$, respectively, for a source at $\delta=35.46^{\circ}$. This corresponds to energy-integrated, all-flavour neutrino luminosity, in the central $90 \%$ energy range, of $\mathcal{L}_{v} \approx 4 \times 10^{48} / 5 \times 10^{47} / 3 \times 10^{46} \mathrm{erg} \mathrm{s}^{-1}$ for a source at $z=0.557$. For a population of neutrino producing sources with summed expectation of order one neutrino, the energy flux estimate given above roughly corresponds to the total energy flux produced by the source population, whereas the individual source contribution, and thus the individual neutrino luminosity, is much lower than our estimate above (IceCube Collaboration 2018a).

\subsection{Swift data}

The Neil Gehrels Swift observatory (Gehrels et al. 2004) observed 3HSP J095507.9+35510137 times; 26 pointings were performed between 2012 and 2013. The remaining pointings were carried out either as a Target of Opportunity (ToO) after the IceCube200107A event, which revealed the source to be in a flaring and very hard state (Giommi et al. 2020b; Krauss et al. 2020) or as part of a subsequent monitoring programme. We analysed all the X-Ray Telescope (XRT; Burrows et al. 2005) imaging data using Swift-DeepSky, a Docker container ${ }^{4}$ encapsulated pipeline software developed in the context of the Open Universe initiative (Giommi et al. 2018, 2019). Spectral analysis was also performed on all exposures with a sufficiently strong signal using the XSPEC-12 software embedded in a dedicated processing pipeline, called Swift-xrtproc, that was first presented in Giommi (2015). Details of the results are given in the appendix. The $2-10 \mathrm{keV}$ emission from 3HSP J095507.9+355101 exhibited over a factor of ten variability in intensity associated with strong spectral changes following a harder-when-brighter trend (see Tables A.1, A.2, and Fig. A.1). The ToO observation of 3HSP J095507.9+355101 found this object in a flaring and hard state, with a 2-10 keV X-ray flux of $\sim 5 \times 10^{-12} \mathrm{erg} \mathrm{cm}^{-2} \mathrm{~s}^{-1}$ a factor 2.5 larger than the average value observed in 2012-2013, and with a power-law spectral index $\Gamma=1.8 \pm 0.06$. A logparabola model gives a similar slope at $1 \mathrm{keV}$ and curvature parameter consistent with zero, implying $v_{\text {peak }}^{S} \gtrsim 2 \times 10^{18} \mathrm{~Hz}$. The optical and UV data of the Ultra-Violet and Optical telescope (UVOT; Roming et al. 2005) were analysed using the on-line tools of the SSDC interactive archive ${ }^{5}$. Spectral data are shown in Fig. 2, while the X-ray light-curve is shown in Fig. 3. The opti$\mathrm{cal} / \mathrm{UV}$ and low-energy X-ray data reach their maximum intensity after the neutrino arrival and remain approximately constant for the subsequent $\sim 30$ days, implying that all the variability in the $2-10 \mathrm{keV}$ band is induced by strong spectral changes above $\sim 7.3 \times 10^{17} \mathrm{~Hz}$.

\footnotetext{
4 https://www . docker. com

http://WWW.ssdc.asi.it
} 

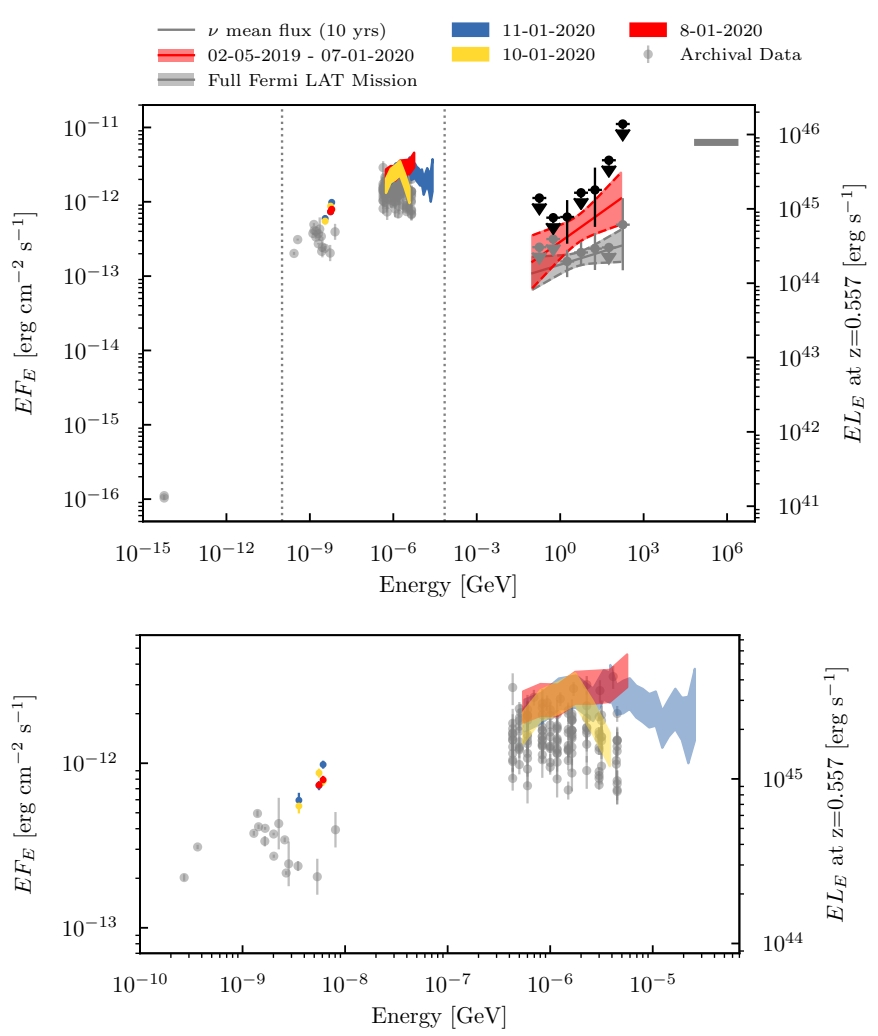

Fig. 2. 3HSP J095507.1+355101 SED. The grey points refer to archival data and, in the case of Fermi-LAT data, the time-integrated measurement up to the neutrino arrival alert. The average all-flavour neutrino flux is shown for an assumed live time of $10 \mathrm{yr}$. Coloured data points are Swift and NUSTAR measurements made around the neutrino arrival time. The black/grey $\gamma$-ray points refer to the red/grey bow ties, indicating the one-sigma uncertainty of the $\gamma$-ray measurement 250 days before the observation of IceCube-200107A and during the full mission, respectively. The best-fit fluxes are shown as solid lines. Upper panel: full hybrid SED, while lower panel: enlarged view of the optical and X-ray bands.

\subsection{NUSTAR data}

3HSP J095507.9+355101 was observed by the NuSTAR hard Xray observatory (Harrison et al. 2013) four days after the detection of IceCube-200107A, following the results of the Swift ToO mentioned above. The observation was partly simultaneous with the third Swift pointing after the neutrino event. The source was detected between $3 \mathrm{keV}$ and $\sim 30 \mathrm{keV}$. A power-law spectral fit gives a best-fit slope of $\Gamma=2.21 \pm 0.06$ with a reduced $\chi_{v}^{2}=0.93$. The data, converted to spectral energy distribution (SED) units, are shown as light blue symbols in Fig. 2.

\subsection{Fermi-LAT data}

The analysis of the $\gamma$-ray emission of 3HSP J095507.9+355101 is based on publicly available Fermi-LAT Pass 8 data acquired in the period August 4, 2008 to January 8, 2020. In order to describe the spectral evolution of the source, we analysed two time windows: the full mission and the last 250 days before the detection of IceCube-200107A. The 250 days are needed to ensure the collection of sufficient photon statistics. The resulting fit between MJD58605.6 and 58855.6 gives evidence for emission with significance of $2.9 \sigma$ and spectral index of $\Gamma=$ $1.73 \pm 0.31$ for a typical single power-law model. The spectral index over the full mission is $\Gamma=1.88 \pm 0.15$, with photon

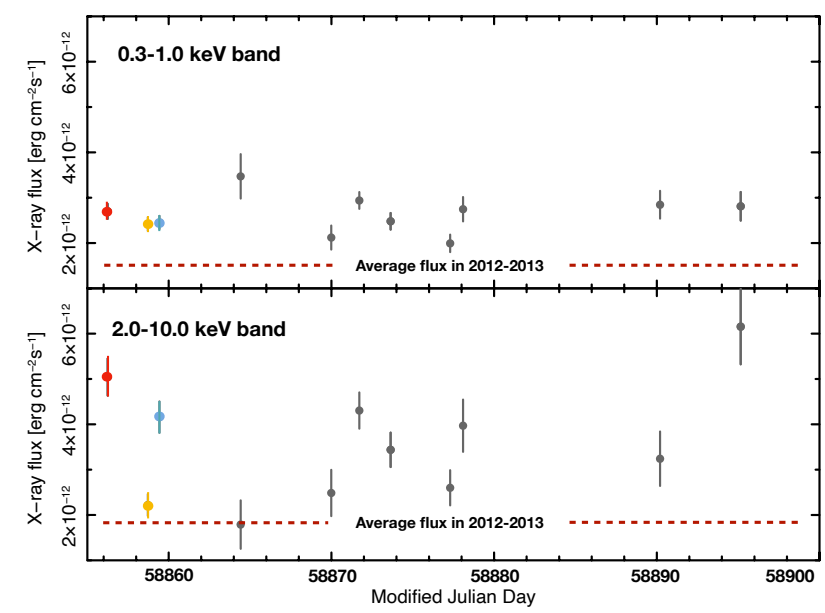

Fig. 3. Swift soft and hard X-ray monitoring of 3HSP J095507.1+355101 after the neutrino arrival. The first observation was carried out one day after the detection of IC200107A. The colours match those used in the SED of Fig. 2. The flux is higher than the average observed in 2012-2013 in both bands, but short-term variability is only present in the $2-10 \mathrm{KeV}$ energy band.

associations up to $178 \mathrm{GeV}$ at $99 \%$ C.L. and a detection significance of $6.3 \sigma$. The corresponding photon fluxes integrated over the entire energy range between $100 \mathrm{MeV}$ and the highest energy photon at $178 \mathrm{GeV}$ are $\left(1.11_{-0.52}^{+0.95}\right) \times 10^{-9} \mathrm{ph} \mathrm{cm}^{-2} \mathrm{~s}^{-1}$ (250 days) and $\left(0.61_{-0.19}^{+0.27}\right) \times 10^{-9} \mathrm{ph} \mathrm{cm}^{-2} \mathrm{~s}^{-1}$ (full mission), respectively. The best-fit spectra are also visualised together with their respective SED points in Fig. 2. More details on the data analysis are given in the appendix.

\subsection{LBT data}

3HSP J095507.9+355101 was observed on January 29, 2020 at the Large Binocular Telescope (LBT; Pogge et al. 2010) in the optical band (4100-8500 $)$. A firm redshift $z=0.557$ was derived thanks to the clear detection of absorption features attributed to its host galaxy. No narrow emission line was detected down to an equivalent width $\sim 0.3 \AA$. This corresponds to [O II] and [O III] line luminosities $<2 \times 10^{40} \mathrm{erg} \mathrm{s}^{-1}$. Details about the spectroscopic study of the source, its host galaxy, and close environment are given in Paiano et al. (2020).

\section{Nature of 3HSP J095507.9+355101}

The SED of 3HSP J095507.9+355101, assembled using multifrequency historical data, shows that this source exhibits a $v_{\text {peak }}^{S} \sim 5 \times 10^{17} \mathrm{~Hz}$ (Chang et al. 2019), which is a very large value that is rarely reached even by extreme blazars (Biteau et al. 2020). The 3HSP catalogue includes only 80 sources with $v_{\text {peak }}^{S} \geq 5 \times 10^{17} \mathrm{~Hz}$ that have been detected by FermiLAT in $\sim 34000$ square degrees of high Galactic latitude sky $\left(|b|>10^{\circ}\right)$, corresponding to an average density of one object every 425 square degrees. The chance probability that one such extreme source is included in the IC 200107A error region of 7.3 square degrees is therefore $7.3 / 425$, or about $1.7 \%$. At the time of the neutrino detection, 3HSP J095507.9+355101 was also found to be in a very hard state $\left(v_{\text {peak }}^{S} \gtrsim 2 \times 10^{18} \mathrm{~Hz}[10 \mathrm{keV}]\right.$ and flaring; see Fig. 2). Blazars are known to spend a small fraction of their time in a very high X-ray state (Giommi et al. 1990). We used the Open Universe blazar database (Giommi 
et al., in prep.) to estimate how frequently the extreme sources with $v_{\text {peak }}^{S} \gtrsim 5 \times 10^{17} \mathrm{~Hz}$, and observed by Swift (in WT or PC mode; Burrows et al. 2005) more than 100 times (MRK421, MRK501, 1ES2344+514, and 1ES0033+595), are detected in a flaring state. We find that they spend less than $10 \%$ of the time at an intensity, that is larger than twice the average value. The overall chance probability of finding a blazar with $v_{\text {peak }}^{S}$ as high as that of 3HSP J095507.9+355101 in the error region of IceCube-200107A during a flaring event is therefore a fraction of $1 \%$. Since this is a posterior estimation based on archival data, which may hide possible biases, it should not be taken as evidence for a firm association, but rather as the identification of an uncommon and physically interesting event that corroborates a persistent trend (e.g. IceCube Collaboration 2018a; Giommi et al. 2020a) and motivates this work. We studied the nature of 3HSP J095507.1+355101, following Padovani et al. (2019), to check if this source is also a masquerading BL Lac like TXS 0506+056, that is intrinsically a flat-spectrum radio quasar (FSRQ) with the emission lines heavily diluted by a strong, Doppler-boosted jet. Given the upper limits on its $L_{\mathrm{O} \text { II }}$ and $L_{\mathrm{O} \text { III }}$ and its black hole mass estimate $\left(M_{\mathrm{BH}} \sim 3 \times 10^{8} M_{\odot}\right.$; Paiano et al. 2020), we obtained the following results for this source: 1. Its radio and $\mathrm{O}$ II luminosities put it at the very edge of the locus of jetted quasars (Fig. 4 of Kalfountzou et al. 2012). 2. Its Eddington ratio is $L / L_{\text {Edd }}<0.02$, which is formally still within the range of high-excitation galaxies (HEGs; characterised by $L / L_{\mathrm{Edd}} \gtrsim 0.01$ ) but barely so. 3 . Its broad-line region (BLR) power in Eddington units is $L_{\mathrm{BLR}} / L_{\mathrm{Edd}}<3 \times 10^{-4}$, which implies that this source is not an FSRQ according to Ghisellini et al. (2011) (as this would require $L_{\mathrm{BLR}} / L_{\mathrm{Edd}} \gtrsim 5 \times 10^{-4}$ ). 4. Finally, its $L_{\gamma} / L_{\text {Edd }}$ values range between $\sim 0.04$ and $\sim 0.10$, depending on its state, that is they straddle the BL Lac - FSRQ division proposed by Sbarrato et al. (2012) $\left(L_{\gamma} / L_{\text {Edd }} \sim 0.1\right)$. Based on all of the above we consider 3HSP J095507.1+355101 an unlikely masquerading BL Lac. Figure 4 shows the location of 3HSP J095507.1+355101 on the $v_{\text {peak }}^{S}$ versus $L_{\gamma}$ plane in its average state and during the flare. The source is an extreme outlier of the so-called blazar sequence, even more so than TXS 0506+056. Given its $L_{\gamma}$, its $v_{\text {peak }}^{S}$ should be about five orders of magnitude smaller to fit the sequence.

\section{Theoretical considerations and conclusions}

We now present some general, model-independent, theoretical constraints on neutrino production by 3HSP J095507.9+355101 based on the multiwavelength observations. A comprehensive overview of models of neutrino emission from $3 \mathrm{HSP}$ J095507.9+355101 is presented in Petropoulou et al. (2020a). Neutrino production in the blazar jet is most likely facilitated by photo-pion $(p \pi)$ interactions. The neutrino production efficiency can thus be parametrised by $f_{p \pi}$, the optical depth to $p \pi$ interactions. Of the energy lost by protons with energy $\varepsilon_{p}$ in $p \pi$ interactions, three-eighths go to neutrinos, resulting in the production of neutrinos with all-flavour luminosity, $\varepsilon_{v} L_{\varepsilon_{v}}=$ $(3 / 8) f_{\mathrm{p} \pi} \varepsilon_{\mathrm{p}} L_{\varepsilon_{\mathrm{p}}}$. Each neutrino is produced with energy $\varepsilon_{v} \approx$ $0.05 \varepsilon_{\mathrm{p}}$. Here and throughout, $\varepsilon L_{\varepsilon}$ is the luminosity per logarithmic energy, $\varepsilon \cdot \mathrm{d} L / \mathrm{d} \varepsilon$, unprimed symbols denote quantities in the cosmic rest frame, quantities with the subscript "obs" refer to the observer frame, and primed quantities refer to the frame co-moving with the jet. Neutrinos produced in interactions with photons co-moving with the jet have typical energy $\varepsilon_{v, \text { obs }} \approx 7.5 \mathrm{PeV}\left(\varepsilon_{\mathrm{t}} / 2 \mathrm{keV}\right)^{-1}(\Gamma / 20)^{2}(1+z)^{-2}$, where $\Gamma$ is the bulk Lorentz factor of the jet, and $\varepsilon_{t}$ the energy of the tar-

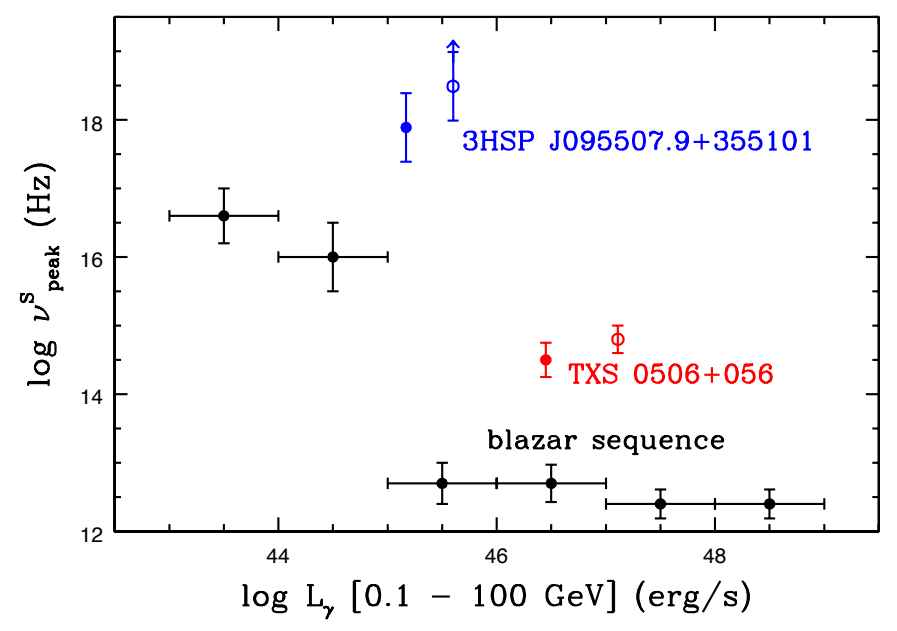

Fig. 4. Rest-frame $v_{\text {peak }}^{S}$ vs. $L_{\gamma}$ for the revised blazar sequence (black points; Ghisellini et al. 2017) and TXS 0506+056 and 3HSP J095507.9+355101. The red and blue points indicate the average [filled] and $\gamma$-ray flare [open] values, respectively. The TXS 0506+056 values are from Padovani et al. (2019). The error bars denote the sample dispersion (blazar sequence) and the uncertainty (TXS 0506+056 and 3HSP J095507.9+355101) respectively.

get photons assuming that protons are accelerated to at least $150 \mathrm{PeV}$.

The remaining five-eighths of the proton energy lost go towards the production of electrons and pionic $\gamma$-rays. Synchrotron emission from electrons/positrons produced in $p \pi$ interactions and two-photon annihilation of the pionic $\gamma$-rays result in the synchrotron cascade flux (Murase et al. 2018) written as

$\left.\varepsilon_{v} L_{\varepsilon_{v}} \approx \frac{6\left(1+Y_{\mathrm{IC}}\right)}{5} \varepsilon_{\gamma} L_{\varepsilon_{\gamma}}\right|_{\varepsilon_{\mathrm{syn}}^{\mathrm{p} \pi}} \approx 8 \times 10^{44} \mathrm{erg} \mathrm{s}^{-1}\left(\frac{\left.\varepsilon_{\gamma} L_{\varepsilon_{\gamma}}\right|_{\varepsilon_{\mathrm{syn}}^{\mathrm{p} \pi}}}{7 \times 10^{44}}\right)$,

where $Y_{\mathrm{IC}}$ is the Compton- $Y$ parameter, typically expected to be $Y_{\mathrm{IC}} \ll 1$ and the $\gamma$-ray emission is expected at energy $\varepsilon_{\text {syn,obs }}^{\mathrm{p} \pi} \approx 39.4 \mathrm{GeV}(B / 0.3 \mathrm{G})\left(\varepsilon_{v, \text { obs }} / 7.5 \mathrm{PeV}\right)^{2}(20 / \delta)(1+$ $z)^{-1}$. The 250 day average luminosity of the flaring SED of 3HSP J095507.9+355101 in the Fermi-LAT energy range thus imposes a limit to the average neutrino luminosity according to Eq. (1). If the neutrino emission lasted 250 days, the expected neutrino luminosity of Eq. (1), is $\sim 2.2$ orders of magnitude lower than the flux implied by the detection of one neutrino according to the estimate of Sect. 2.1, which is $\varepsilon_{v} L_{\varepsilon_{v}}=$ $\mathcal{L}_{v} / \ln (2.6 \mathrm{PeV} / 65 \mathrm{TeV}) \approx 1.3 \times 10^{47} \mathrm{erg} \mathrm{s}^{-1}$. The expected neutrino luminosity as a function of the proton luminosity is shown in Fig. 5, for two characteristic values of $f_{\mathrm{p} \pi}$ (by definition $f_{\mathrm{p} \pi} \leq 1$ ), together with the constraint imposed by Eq. (1) and the luminosity needed to produce 1 neutrino in IceCube. Figure 5 also gives the "baryon loading" factor, $\xi$, implied by a given proton luminosity, defined in this work as $\xi=\varepsilon_{\mathrm{p}} L_{\varepsilon_{\mathrm{p}}} / \varepsilon_{\gamma} L_{\varepsilon_{\gamma}}{ }^{6}$. Considering the long-term average Fermi-LAT flux instead, Eq. (1) leads to an upper limit on $\left.\varepsilon_{v} L_{\varepsilon_{v}} \approx\left[6 /\left(1+Y_{\mathrm{IC}}\right) 5\right] \varepsilon_{\gamma} L_{\varepsilon_{\gamma}}\right|_{\varepsilon_{\mathrm{syn}}^{\mathrm{p} \pi}} \approx 3 \times$ $10^{44} \mathrm{erg} \mathrm{s}^{-1}$. This is a factor of $\sim 30$ lower than the neutrino luminosity needed to detect 1 neutrino in IceCube, assuming a $10 \mathrm{yr}$ live time, which is $\mathcal{L}_{v} / \ln (2.6 \mathrm{PeV} / 65 \mathrm{TeV}) \approx 8 \times 10^{45} \mathrm{erg} \mathrm{s}^{-1}$. Thus, if the neutrino emission was related to the long-term emission of 3HSP J095507.9+355101, it is easier to satisfy the

6 We approximated $\varepsilon_{\gamma} L_{\varepsilon_{\gamma}} \sim \mathcal{L}_{\gamma} / \ln (320 \mathrm{GeV} / 100 \mathrm{MeV})$, where $\mathcal{L}_{\gamma}=$ $5.66 \times 10^{45} \mathrm{erg} \mathrm{s}^{-1}$ is the $\gamma$-ray luminosity measured with the Fermi-LAT during the 250 day flare. 


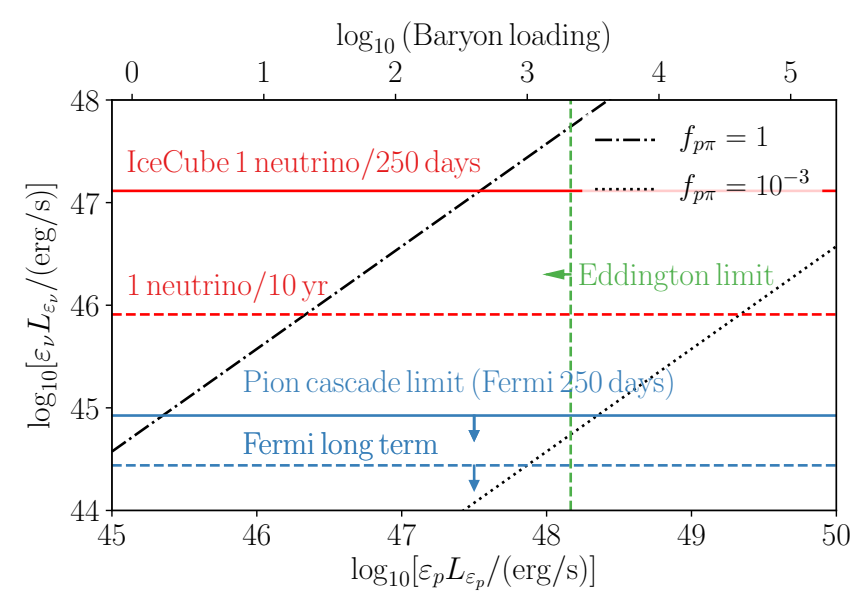

Fig. 5. All-flavour neutrino luminosity as a function of proton luminosity for two different values of the optical depth to photopion interactions $f_{\mathrm{p} \pi}$. The red solid (dashed) line gives the neutrino luminosity corresponding to 1 muon neutrino in IceCube from 3HSP J095507.9+355101 if the neutrino emission lasted 250 days (10 yr). The blue horizontal solid (dashed) line gives the upper limit to the neutrino luminosity implied by the Fermi-LAT 250-day (long-term average) spectrum. The green line shows the upper limit to the proton luminosity implied by the Eddington luminosity of the $3 \times 10^{8} M_{\odot}$ black hole, assuming $\Gamma=20$, proton spectral index -2 , and maximum proton energy $10^{18} \mathrm{eV}$.

$\gamma$-ray emission constraint than if the neutrino emission was related to the Fermi-LAT 250-day high state. These results are also summarised in Fig. 5. Below the threshold for $p \pi$ interactions, protons lose energy via the Bethe-Heitler $(\mathrm{BH})$ process. Unlike in the case of TXS 0506+056, for 3HSP J095507.9+ 355101 there are no observations available to constrain the $\mathrm{BH}$ cascade component and the most stringent constraint on the neutrino luminosity comes from the $p \pi$ cascade. Figure 5 reveals the difficulty of canonical theoretical models to explain the observation of one neutrino from 3HSP J095507.9+355101 during the 250 day Fermi high state, and to a lesser extent during the $10 \mathrm{yr}$ of IceCube observations. The Poisson probability to detect one neutrino is $\sim 0.01$ and $\sim 0.03$ for the two timescales, respectively, which could be interpreted as a statistical fluctuation to account for the association. We note that a similar neutrino luminosity upper limit has been derived in one-zone models of neutrino production of TXS 0506+056 during its 2017 flare (Ansoldi et al. 2018; Cerruti et al. 2019; Gao et al. 2019; Keivani et al. 2018; Petropoulou et al. 2020b), which must also be interpreted as an upward $(\sim 2 \sigma)$ fluctuation to account for the observed association. On the other hand, Eq. (1) assumes that neutrinos and $\gamma$-rays are co-spatially emitted. In the presence of multiple emitting zones, and/or an obscuring medium for the $\gamma$-rays, the constraint of Eq. (1) can be relaxed and larger neutrino luminosity may be produced by 3HSP J095507.9+355101 (see for example such models for the 2017 flare of TXS 0506+056: Murase et al. 2018; Liu et al. 2019; Oikonomou et al. 2019; Xue et al. 2019; Zhang et al. 2020).

In summary, 3HSP J095507.9+355101, with its extremely high $v_{\text {peak }}^{S}$ is the second IBL/HBL that is off the blazar sequence to be detected in the error region of a high-energy neutrino during a flare. The Eddington ratio and upper limit to the BLR power we obtained make 3HSP J095507.9+355101 an unlikely masquerading BL Lac, in contrast to TXS 0506+056; this new observation points to a different class of possible neutrinoemitting BL Lac objects, which do not possess a (hidden) powerful BLR but have abundant $>\mathrm{keV}$ photons, owing to the high $v_{\text {peak }}^{S}$, which may facilitate $\mathrm{PeV}$ neutrino production. As was the case with TXS $0506+056$, a possible association points to non-standard ("one-zone") theoretical models, and/or the existence of an underlying population of sources each expected to produce $\ll 1$ neutrinos in IceCube but with summed expectation $\geq 1$. Figure 5 reveals that $\sim 150$ (30) sources identical to 3HSP J095507.9+355101 are needed to produce one neutrino in 250 days ( 10 years), corresponding to an expectation of $\lesssim 0.01$ neutrinos from a single blazar of this type. This would imply that the IceCube sensitivity is still above the expected fluxes from similar individual blazars, and the currently observed neutrino counting, if due to blazars, must be driven by large statistical fluctuations. A possible way to reconcile observations with expectations is to consider that there are about 100 catalogued blazars with properties similar to 3HSP J095507.1+355101. If each of these objects emits an average flux of $\sim 0.01$ neutrinos in the period considered, we would be in a situation of extremely low counting statistics where the probability of observing one neutrino from a specific blazar is of the order of $1 \%$. Collectively, however, one neutrino would be expected on similar timescales from one of the $\sim 100$ randomly distributed blazars in the underlying population. This scenario is consistent with the current situation where only single neutrino events from each candidate counterparts are observed. Examples supporting this view are the extreme blazars 3HSP J023248.6+201717, 3HSP J144656.8-265658, and 3HSP J094620.2+010452, which are located inside the $90 \%$ uncertainty region of IC $111216 \mathrm{~A}$, IC 170506A, and IC 190819 (Giommi et al. 2020a).

Acknowledgements. We acknowledge the use of data and software facilities from the ASI-SSDC and the tools developed within the United Nations "Open Universe" initiative. This work is supported by the Deutsche Forschungsgemeinschaft through grant SFB 1258 "Neutrinos and Dark Matter in Astro and Particle Physics". We thank Riccardo Middei for his help with the analysis of NuSTAR data. We thank Matthias Huber and Michael Unger for useful discussions on the interpretation of the IceCube observations.

\section{References}

Aartsen, M. G., Abraham, K., Ackermann, M., et al. 2017, ApJ, 835, 45 Abdo, A. A., Ackermann, M., Agudo, I., et al. 2010, ApJ, 716, 30

Ansoldi, S., Antonelli, L. A., Arcaro, C., et al. 2018, ApJ, 863, L10 Biteau, J., Prandini, E., Costamante, L., et al. 2020, Nat. Astron., 4, 124 Blaufuss, E., Kintscher, T., Lu, L., \& Tung, C. F. 2020, PoS ICRC2019, 1021 Burrows, D. N., Hill, J. E., Nousek, J. A., et al. 2005, Space Sci. Rev., 120, 165 Cerruti, M., Zech, A., Boisson, C., et al. 2019, MNRAS, 483, L12

Chang, Y. L., Arsioli, B., Giommi, P., Padovani, P., \& Brandt, C. H. 2019, A\&A, 632, A77

Gao, S., Fedynitch, A., Winter, W., \& Pohl, M. 2019, Nat. Astron., 3, 88

Gehrels, N., Chincarini, G., Giommi, P., et al. 2004, ApJ, 611, 1005

Ghisellini, G., Tavecchio, F., Foschini, L., \& Ghirlanda, G. 2011, MNRAS, 414, 2674

Ghisellini, G., Righi, C., Costamante, L., \& Tavecchio, F. 2017, MNRAS, 469, 255

Giommi, P. 2015, J. High Energy Astrophys., 7, 173

Giommi, P., Barr, P., Garilli, B., Maccagni, D., \& Pollock, A. M. T. 1990, ApJ, 356,432

Giommi, P., Arrigo, G., Barres De Almeida, U., et al. 2018, ArXiv e-prints [arXiv:1805.08505]

Giommi, P., Brandt, C. H., Barres de Almeida, U., et al. 2019, A\&A, 631, A116 Giommi, P., Glauch, T., Padovani, P., et al. 2020a, MNRAS, in press [arXiv:2001.09355]

Giommi, P., Glauch, T., \& Resconi, E. 2020b, ATel, 13394, 1

Harrison, F. A., Craig, W. W., Christensen, F. E., et al. 2013, ApJ, 770, 103

IceCube Collaboration 2014, Phys. Rev. Lett., 113, 101101

IceCube Collaboration 2017a, ArXiv e-prints [arXiv:1710.01191]

IceCube Collaboration 2017b, ArXiv e-prints [arXiv:1710.01179]

IceCube Collaboration 2018a, Science, 361, eaat 1378

IceCube Collaboration 2018b, Science, 361, 147 
Kalfountzou, E., Jarvis, M. J., Bonfield, D. G., \& Hardcastle, M. J. 2012, MNRAS, 427, 2401

Keivani, A., Murase, K., Petropoulou, M., et al. 2018, ApJ, 864, 84

Krauss, F., Gregoire, T., Fox, D. B., Kennea, J., \& Evans, P. 2020, ATel, 13395 1

Kronmueller, M., \& Glauch, T. 2020, PoS ICRC2019, 937

Liu, R.-Y., Wang, K., Xue, R., et al. 2019, Phys. Rev. D, 99, 063008

Lucarelli, F., Tavani, M., Piano, G., et al. 2019, ApJ, 870, 136

Massaro, E., Perri, M., Giommi, P., \& Nesci, R. 2004, A\&A, 413, 489

Murase, K., Oikonomou, F., \& Petropoulou, M. 2018, ApJ, 865, 124

Oikonomou, F., Murase, K., Padovani, P., Resconi, E., \& Mészáros, P. 2019 MNRAS, 489, 4347

Padovani, P., \& Giommi, P. 1995, ApJ, 444, 567

Padovani, P., \& Resconi, E. 2014, MNRAS, 443, 474

Padovani, P., Resconi, E., Giommi, P., Arsioli, B., \& Chang, Y. L. 2016, MNRAS, 457, 3582

Padovani, P., Giommi, P., Resconi, E., et al. 2018, MNRAS, 480, 192

Padovani, P., Oikonomou, F., Petropoulou, M., Giommi, P., \& Resconi, E. 2019, MNRAS, 484, L104
Paiano, S., Falomo, R., Treves, A., \& Scarpa, R. 2018, ApJ, 854, L32 Paiano, S., Falomo, R., Padovani, P., et al. 2020, MNRAS, 495, L108 Petropoulou, M., Oikonomou, F., Mastichiadis, A., et al. 2020a, ApJ, submitted [arXiv:2005.07218]

Petropoulou, M., Murase, K., Santander, M., et al. 2020b, ApJ, 891, 115

Pizzuto, A. 2020, GRB Coordinates Network, 26704, 1

Pogge, R. W., Atwood, B., Brewer, D. F., et al. 2010, Proc. SPIE, 7735, 77350A

Resconi, E., Coenders, S., Padovani, P., Giommi, P., \& Caccianiga, L. 2017, MNRAS, 468, 597

Roming, P. W. A., Kennedy, T. E., Mason, K. O., et al. 2005, Space Sci. Rev., 120,95

Sbarrato, T., Ghisellini, G., Maraschi, L., \& Colpi, M. 2012, MNRAS, 421, 1764

Schneider, A. 2020, PoS ICRC2019, 1004

Stein, R. 2020, GRB Coordinates Network, 26655, 1

Stettner, J. 2020, PoS ICRC2019, 1017

Xue, R., Liu, R.-Y., Petropoulou, M., et al. 2019, ApJ, 886, 23

Zhang, B. T., Petropoulou, M., Murase, K., \& Oikonomou, F. 2020, ApJ, 889, 118 


\section{Appendix A}

In this appendix we give details of the multi-frequency data analysis of 3HSP J095507.1+355101.

\section{A.1. Swift-XRT}

All Swift-XRT observations were analysed using Swift-DeepSky and Swift-xrtproc, the imaging and spectral analysis tools developed within the Open Universe initiative (Giommi et al. 2019; Giommi 2015). Both tools are based on the official HEASoft data reduction package, in particular on XIMAGE-4.5 and XSPEC12; these tools are particularly useful when analysing a large number of observations, as the tools automatically download the data and calibration files from one of the official archives, generate all the necessary intermediate products, and conduct a detailed standard analysis. The results of the image analysis are presented in Table A.1, in which Col. 1 gives the observation start time, Col. 2 gives the effective exposure time, Col. 3 gives the count rate in the $0.3-10 \mathrm{keV}$ band, and Cols. $4-7$ give the flux in the $0.3-10,0.3-1.0,1-2 \mathrm{keV}$, and $2-10 \mathrm{KeV}$ bands, respectively. The largest flux variations are observed in the $2-10 \mathrm{keV}$ band where the intensity varied by over a factor ten, between a minimum of $0.55 \times 10^{-12} \mathrm{erg} \mathrm{cm}^{-2} \mathrm{~s}^{-1}$ on MJD 56233 (Nov. 2, 2012) and a maximum of $6.16 \times 10^{-12} \mathrm{erg} \mathrm{cm}^{-2} \mathrm{~s}^{-1}$ on MJD 58900 (Feb. 21, 2020).

Details of the spectral analysis for the cases of powerlaw and log parabola models with NH fixed to the Galactic value, are given in Table A.2. Column 1 gives the observation date, Col. 2 gives the best fit photon spectral index with one $\sigma$ error, Col. 3 gives the value of the reduced $\chi^{2}$ with the number of degrees of freedom (d.o.f.) in parenthesis, Cols. 4 and 5 give the spectral slope at $1 \mathrm{keV}(\alpha)$ and curvature parameter $(\beta)$ with one $\sigma$ error, and Col. 6 the corresponding reduced $\chi^{2}$ and d.o.f. Figure A. 1 shows the best-fit power-law spectral index versus the $2-10 \mathrm{keV}$ flux, for all the observations where the error on the spectral slope is smaller than 0.25 . The figure shows a clear harder-when-brighter trend, a behaviour seen in several other HBL blazars (e.g. Giommi et al. 1990).

Table A.1. Results of the imaging analysis of all Swift-XRT observations of 3HSP J095507.1+355101 with exposure time larger than $200 \mathrm{~s}$.

\begin{tabular}{|c|c|c|c|c|c|c|}
\hline $\begin{array}{l}\text { Observation } \\
\text { start time } \\
\text { MJD } \\
(1)\end{array}$ & $\begin{array}{c}\text { Exposure } \\
\text { time } \\
\mathrm{s} \\
(2) \\
\end{array}$ & $\begin{array}{c}\text { Count rate } \\
0.3-10 \mathrm{keV} \\
\text { cts s }^{-1} \\
(3)\end{array}$ & $\begin{array}{c}\text { Flux } \\
0.3-10 \mathrm{keV} \\
10^{-12} \mathrm{erg} \mathrm{cm}^{-2} \mathrm{~s}^{-1} \\
(4)\end{array}$ & $\begin{array}{c}\text { Flux } \\
0.3-1 \mathrm{keV} \\
10^{-12} \underset{(5)}{\mathrm{erg} \mathrm{cm}^{-2} \mathrm{~s}^{-1}} \\
\end{array}$ & $\begin{array}{c}\text { Flux } \\
1-2 \mathrm{keV}^{2} \\
10^{-12} \mathrm{erg} \mathrm{cm}^{-2} \mathrm{~s}^{-1} \\
(6)\end{array}$ & $\begin{array}{c}\text { Flux } \\
2-10 \mathrm{keV}^{-1} \\
10^{-12} \mathrm{erg} \mathrm{cm}^{-2} \mathrm{~s}^{-1} \\
(7)\end{array}$ \\
\hline 56036.1523 & 845. & $0.13 \pm 0.01$ & $4.21 \pm 0.42$ & $1.36 \pm 0.20$ & $0.83 \pm 0.14$ & $2.04 \pm 0.45$ \\
\hline 56208.1641 & 4296. & $0.12 \pm 0.01$ & $4.09 \pm 0.18$ & $1.14 \pm 0.08$ & $0.88 \pm 0.06$ & $2.04 \pm 0.20$ \\
\hline 56210.0352 & 3915. & $0.14 \pm 0.01$ & $4.30 \pm 0.19$ & $1.44 \pm 0.10$ & $0.89 \pm 0.07$ & $1.95 \pm 0.20$ \\
\hline 56211.8359 & 232. & $0.14 \pm 0.03$ & $4.81 \pm 0.89$ & $1.48 \pm 0.40$ & $1.05 \pm 0.29$ & $1.02 \pm 0.60$ \\
\hline 56212.6406 & 2074. & $0.14 \pm 0.01$ & $4.10 \pm 0.25$ & $1.50 \pm 0.13$ & $0.92 \pm 0.09$ & $1.63 \pm 0.24$ \\
\hline 56216.0508 & 1559. & $0.10 \pm 0.01$ & $3.03 \pm 0.25$ & $1.04 \pm 0.13$ & $0.72 \pm 0.10$ & $1.21 \pm 0.25$ \\
\hline 56217.1172 & 564. & $0.09 \pm 0.01$ & $3.14 \pm 0.46$ & $0.89 \pm 0.21$ & $0.56 \pm 0.14$ & $1.45 \pm 0.48$ \\
\hline 56227.0586 & 1447. & $0.12 \pm 0.01$ & $3.61 \pm 0.29$ & $1.33 \pm 0.15$ & $0.76 \pm 0.10$ & $1.48 \pm 0.27$ \\
\hline 56230.9336 & 435. & $0.12 \pm 0.02$ & $4.34 \pm 0.64$ & $0.94 \pm 0.24$ & $1.14 \pm 0.23$ & $1.44 \pm 0.57$ \\
\hline 56233.0039 & 656. & $0.14 \pm 0.01$ & $4.81 \pm 0.53$ & $1.66 \pm 0.25$ & $1.01 \pm 0.17$ & $0.55 \pm 0.28$ \\
\hline 56253.3320 & 809. & $0.14 \pm 0.01$ & $4.00 \pm 0.40$ & $2.07 \pm 0.27$ & $0.60 \pm 0.12$ & $1.37 \pm 0.36$ \\
\hline 56254.3320 & 1892. & $0.08 \pm 0.01$ & $2.39 \pm 0.21$ & $0.92 \pm 0.11$ & $0.44 \pm 0.07$ & $1.02 \pm 0.21$ \\
\hline 56284.6445 & 1374. & $0.14 \pm 0.01$ & $3.96 \pm 0.31$ & $1.73 \pm 0.19$ & $0.96 \pm 0.12$ & $1.16 \pm 0.26$ \\
\hline 56290.2461 & 609. & $0.12 \pm 0.01$ & $4.45 \pm 0.53$ & $1.54 \pm 0.26$ & $0.78 \pm 0.16$ & $1.20 \pm 0.41$ \\
\hline 56298.0039 & 1079. & $0.21 \pm 0.01$ & $6.10 \pm 0.41$ & $2.26 \pm 0.22$ & $1.46 \pm 0.17$ & $2.25 \pm 0.39$ \\
\hline 56305.0898 & 711. & $0.19 \pm 0.02$ & $5.73 \pm 0.50$ & $2.00 \pm 0.26$ & $1.39 \pm 0.18$ & $2.22 \pm 0.48$ \\
\hline 56318.9023 & 1072. & $0.20 \pm 0.01$ & $6.17 \pm 0.43$ & $2.38 \pm 0.23$ & $1.17 \pm 0.14$ & $2.60 \pm 0.42$ \\
\hline 56321.9062 & 583. & $0.17 \pm 0.02$ & $5.93 \pm 0.64$ & $1.70 \pm 0.28$ & $0.93 \pm 0.18$ & $3.39 \pm 0.69$ \\
\hline 56324.5117 & 346. & $0.18 \pm 0.02$ & $6.47 \pm 0.85$ & $1.35 \pm 0.31$ & $1.38 \pm 0.27$ & $3.74 \pm 0.96$ \\
\hline 56329.4531 & 1027. & $0.17 \pm 0.01$ & $5.47 \pm 0.42$ & $1.80 \pm 0.22$ & $1.06 \pm 0.14$ & $2.61 \pm 0.45$ \\
\hline 56332.8594 & 1051. & $0.14 \pm 0.01$ & $4.49 \pm 0.37$ & $1.54 \pm 0.20$ & $0.95 \pm 0.13$ & $1.98 \pm 0.38$ \\
\hline 56334.9922 & 222. & $0.18 \pm 0.03$ & $6.34 \pm 1.07$ & $2.05 \pm 0.49$ & $1.07 \pm 0.31$ & $2.36 \pm 0.97$ \\
\hline 58856.2461 & 2681. & $0.28 \pm 0.01$ & $9.53 \pm 0.37$ & $2.70 \pm 0.17$ & $1.83 \pm 0.12$ & $5.04 \pm 0.41$ \\
\hline 58858.7188 & 2523. & $0.24 \pm 0.01$ & $6.79 \pm 0.29$ & $2.42 \pm 0.15$ & $1.88 \pm 0.11$ & $2.22 \pm 0.24$ \\
\hline 58859.4336 & 2614. & $0.26 \pm 0.01$ & $8.39 \pm 0.33$ & $2.45 \pm 0.15$ & $1.74 \pm 0.11$ & $4.16 \pm 0.35$ \\
\hline 58864.4219 & 373. & $0.30 \pm 0.03$ & $8.03 \pm 0.78$ & $3.47 \pm 0.49$ & $2.31 \pm 0.33$ & $1.79 \pm 0.54$ \\
\hline 58869.9922 & 721. & $0.21 \pm 0.02$ & $6.26 \pm 0.54$ & $2.12 \pm 0.27$ & $1.52 \pm 0.20$ & $2.49 \pm 0.51$ \\
\hline 58871.7148 & 2328. & $0.29 \pm 0.01$ & $9.21 \pm 0.38$ & $2.94 \pm 0.19$ & $1.92 \pm 0.13$ & $4.30 \pm 0.40$ \\
\hline 58873.6406 & 1718. & $0.24 \pm 0.01$ & $7.57 \pm 0.38$ & $2.48 \pm 0.18$ & $1.60 \pm 0.13$ & $3.44 \pm 0.38$ \\
\hline 58877.2969 & 1342. & $0.21 \pm 0.01$ & $6.40 \pm 0.39$ & $1.99 \pm 0.19$ & $1.64 \pm 0.16$ & $2.60 \pm 0.39$ \\
\hline 58878.0898 & 970. & $0.23 \pm 0.02$ & $7.76 \pm 0.53$ & $2.75 \pm 0.27$ & $1.16 \pm 0.15$ & $3.97 \pm 0.58$ \\
\hline 58890.1992 & 724. & $0.25 \pm 0.02$ & $7.72 \pm 0.58$ & $2.84 \pm 0.31$ & $1.58 \pm 0.20$ & $3.24 \pm 0.60$ \\
\hline 58895.1562 & 739. & $0.33 \pm 0.02$ & $11.22 \pm 0.76$ & $2.81 \pm 0.32$ & $2.27 \pm 0.25$ & $6.16 \pm 0.83$ \\
\hline 58900.0117 & 1978. & $0.28 \pm 0.01$ & $9.12 \pm 0.39$ & $2.83 \pm 0.19$ & $1.88 \pm 0.13$ & $4.39 \pm 0.41$ \\
\hline
\end{tabular}




\section{A.2. NUSTAR}

Data from the NUSTAR observation made shortly after the neutrino arrival were analysed using the XSPEC12 package. Photons detected by both telescopes (module A and B) were combined and fitted to spectral models following the standard XSPEC procedure. The source was detected between $3 \mathrm{keV}$ and $30 \mathrm{keV}$. A power-law spectral model gives a best-fit slope of $\Gamma=2.21 \pm 0.06$ with a reduced $\chi_{v}^{2}=0.93$ with 101 d.o.f. A fit to a log parabola model does not improve the reduced $\chi_{v}^{2}$ and therefore it is not reported here. A combined fit of the NuSTAR and the quasi-simultaneous Swift-XRT data with a log parabola model gives the following best-fit parameters: $\alpha=1.80 \pm 0.07$, $\beta=0.24 \pm 0.05$ for a pivot energy $E_{\text {pivot }}=1 \mathrm{keV}$, and reduced $\chi_{v}^{2}=0.86$ with 129 d.o.f. The corresponding SED peak energy, estimated as $E_{\text {peak }}=10^{(2-\alpha) / 2 \beta}$ (Massaro et al. 2004), is $E_{\text {peak }} \sim$ $2.6 \mathrm{keV}$.

\section{A.3. Fermi}

For the analysis of the $\gamma$-ray emission of 3HSP J095507.9+355101 we used the publicly available Fermi-LAT Pass 8 data acquired in the period August 4, 2008 to January 8, 2020 and followed the standard procedure as described in the Fermi cicerone ${ }^{7}$. We constructed a model that contains all known 4FGL sources plus the diffuse Galactic and isotropic emissions. In the likelihood fits, we left free the normalisation and spectral index of all sources within $10^{\circ}$ (corresponding to the $95 \%$ Fermi pointspread function at $100 \mathrm{MeV}$ ). To calculate an a priori estimate of the required integration time for a significant detection of the source, we used the time-integrated measurement in the Fermi 4FGL catalogue. Assuming a signal dominated counting experiment with $\chi_{1}^{2}$ background test-statistic distribution we know that the median test statistic distribution scales linearly in time $t$, i.e., $\mathcal{T S} \propto t$ and, therefore,

Table A.2. Results of the spectral analysis of all Swift-XRT observations of 3HSP J095507.1+355101 with at least 25 net counts.

\begin{tabular}{|c|c|c|c|c|c|}
\hline Observation date & $\begin{array}{c}\text { Power law } \\
\Gamma \\
(2)\end{array}$ & Reduced $\chi^{2}$ & $\begin{array}{c}\text { Log parabola } \\
\alpha \\
(4) \\
\end{array}$ & $\begin{array}{c}\text { Log parabola } \\
\beta \\
(5) \\
\end{array}$ & $\begin{array}{c}\text { Reduced } \chi^{2} \\
\text { (6) }\end{array}$ \\
\hline 56036.1523 & $2.09 \pm 0.15$ & $1.35(75)$ & $2.23 \pm 0.19$ & $-0.48 \pm 0.39$ & $1.25(74)$ \\
\hline 56208.1641 & $1.84 \pm 0.07$ & $1.15(227)$ & $1.75 \pm 0.10$ & $0.30 \pm 0.21$ & $1.12(226)$ \\
\hline 56210.0352 & $1.94 \pm 0.08$ & $0.86(212)$ & $1.87 \pm 0.10$ & $0.28 \pm 0.24$ & $0.83(211)$ \\
\hline 56211.8359 & $2.17 \pm 0.38$ & $0.69(25)$ & $1.97 \pm 0.41$ & $1.58 \pm 1.20$ & $0.55(24)$ \\
\hline 56212.6406 & $1.96 \pm 0.10$ & $1.10(155)$ & $1.92 \pm 0.13$ & $0.15 \pm 0.29$ & $1.12(154)$ \\
\hline 56216.0508 & $1.99 \pm 0.18$ & $0.97(79)$ & $1.71 \pm 0.24$ & $1.15 \pm 0.57$ & $0.83(78)$ \\
\hline 56217.1172 & $2.03 \pm 0.25$ & $0.77(35)$ & $1.99 \pm 0.32$ & $0.15 \pm 0.71$ & $0.79(34)$ \\
\hline 56227.0586 & $2.02 \pm 0.14$ & $1.11(103)$ & $1.89 \pm 0.17$ & $0.63 \pm 0.50$ & 1.03 (102) \\
\hline 56230.9336 & $2.17 \pm 0.27$ & $0.72(39)$ & $2.00 \pm 0.30$ & $0.75 \pm 0.81$ & $0.73(38)$ \\
\hline 56233.0039 & $2.51 \pm 0.21$ & $1.01(63)$ & $2.48 \pm 0.22$ & $0.52 \pm 0.73$ & $1.06(62)$ \\
\hline 56253.3320 & $2.40 \pm 0.17$ & $0.94(77)$ & $2.44 \pm 0.20$ & $-0.25 \pm 0.54$ & $0.93(76)$ \\
\hline 56254.3320 & $2.06 \pm 0.14$ & $0.62(90)$ & $2.15 \pm 0.18$ & $-0.36 \pm 0.42$ & $0.59(89)$ \\
\hline 56284.6445 & $2.16 \pm 0.14$ & $0.90(114)$ & $1.98 \pm 0.16$ & $0.92 \pm 0.45$ & 0.88 (113) \\
\hline 56290.2461 & $2.22 \pm 0.21$ & $1.12(58)$ & $2.08 \pm 0.24$ & $0.75 \pm 0.74$ & $1.23(57)$ \\
\hline 56298.0039 & $2.29 \pm 0.12$ & $1.19(123)$ & $2.26 \pm 0.13$ & $0.13 \pm 0.39$ & 1.19 (122) \\
\hline 56305.0898 & $2.19 \pm 0.14$ & $0.75(97)$ & $2.18 \pm 0.18$ & $4.47 \pm 0.38$ & $0.76(96)$ \\
\hline 56318.9023 & $2.03 \pm 0.12$ & 0.94 (132) & $1.92 \pm 0.15$ & $0.38 \pm 0.35$ & $0.95(131)$ \\
\hline 56321.9062 & $1.73 \pm 0.16$ & $0.86(76)$ & $1.74 \pm 0.28$ & $-2.91 \pm 0.53$ & $0.87(75)$ \\
\hline 56324.5117 & $1.76 \pm 0.22$ & 0.77 (47) & $1.73 \pm 0.28$ & $9.21 \pm 0.74$ & $0.78(46)$ \\
\hline 56329.4531 & $2.00 \pm 0.13$ & $0.84(113)$ & $1.94 \pm 0.17$ & $0.21 \pm 0.39$ & 0.85 (112) \\
\hline 56332.8594 & $1.89 \pm 0.14$ & $1.27(96)$ & $1.93 \pm 0.18$ & $-0.16 \pm 0.44$ & $1.28(95)$ \\
\hline 56334.9922 & $1.99 \pm 0.29$ & $0.74(30)$ & $2.11 \pm 0.31$ & $-0.83 \pm 0.98$ & $0.75(29)$ \\
\hline 58856.2461 & $1.77 \pm 0.06$ & $1.04(274)$ & $1.70 \pm 0.09$ & $0.20 \pm 0.18$ & $1.04(273)$ \\
\hline 58858.7188 & $1.98 \pm 0.07$ & $1.51(216)$ & $1.76 \pm 0.10$ & $0.76 \pm 0.21$ & $1.37(215)$ \\
\hline 58859.4336 & $1.81 \pm 0.06$ & $0.90(254)$ & $1.75 \pm 0.09$ & $0.18 \pm 0.19$ & $0.89(253)$ \\
\hline 58864.4219 & $2.06 \pm 0.21$ & $1.00(70)$ & $1.80 \pm 0.23$ & $1.56 \pm 0.69$ & $0.96(69)$ \\
\hline 58869.9922 & $1.97 \pm 0.13$ & $1.22(101)$ & $1.94 \pm 0.17$ & $8.95 \pm 0.38$ & $1.23(100)$ \\
\hline 58871.7148 & $1.92 \pm 0.07$ & $1.21(244)$ & $1.88 \pm 0.09$ & $0.15 \pm 0.19$ & $1.20(243)$ \\
\hline 58873.6406 & $1.97 \pm 0.08$ & $0.93(188)$ & $1.97 \pm 0.11$ & $-1.50 \pm 0.25$ & 0.94 (187) \\
\hline 58877.2969 & $1.87 \pm 0.11$ & $1.02(145)$ & $1.71 \pm 0.15$ & $0.49 \pm 0.31$ & $0.95(144)$ \\
\hline 58878.0898 & $1.98 \pm 0.12$ & 1.04 (124) & $1.88 \pm 0.16$ & $0.34 \pm 0.36$ & $1.05(123)$ \\
\hline 58890.1992 & $2.00 \pm 0.14$ & $1.21(113)$ & $1.80 \pm 0.19$ & $0.93 \pm 0.50$ & $1.08(112)$ \\
\hline 58895.1562 & $1.78 \pm 0.12$ & $0.81(141)$ & $1.55 \pm 0.16$ & $0.69 \pm 0.36$ & $0.76(140)$ \\
\hline 58900.0117 & $1.93 \pm 0.07$ & $1.14(219)$ & $1.84 \pm 0.10$ & $0.28 \pm 0.21$ & $1.13(218)$ \\
\hline
\end{tabular}

\footnotetext{
7 https://fermi.gsfc.nasa.gov/ssc/data/analysis/ documentation/Cicerone/
} 


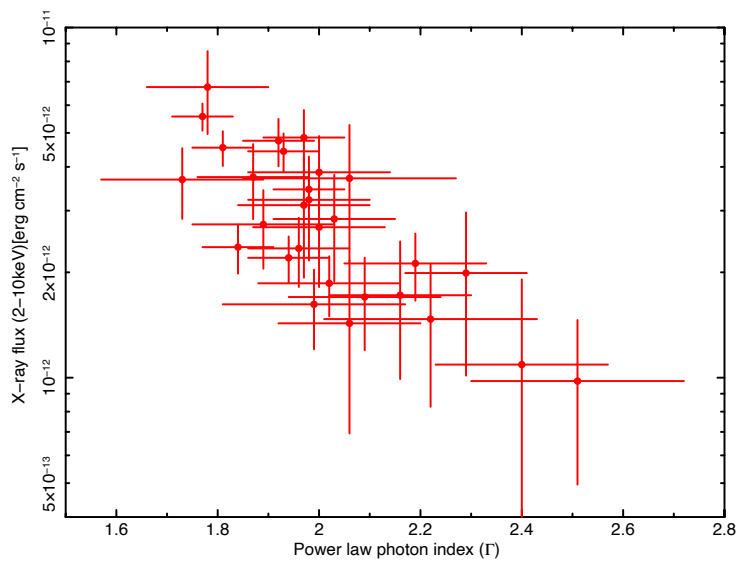

Fig. A.1. Best-fit power-law spectral index of all the observations with errors smaller than 0.25 is plotted vs. the $2-10 \mathrm{keV} \mathrm{X}$-ray flux of 3HSP J095507.1+355101.

$t_{\mathrm{lc}}=\left(\mathcal{T} \mathcal{S}_{\mathrm{lc}} / \mathcal{T} \mathcal{S}_{2920 \mathrm{~d}}\right) \cdot 2920[$ days $]$

assuming a quasi-steady emission. Here $\mathcal{T} \mathcal{S}_{\text {lc }}$ defines the target test-statistic value with required integration time $t_{\mathrm{lc}}$. 2920 days
Table A.3. Energy dependent significance of the Fermi-LAT SED for the full-mission and the 250 days before the neutrino alert.

\begin{tabular}{lcc}
\hline \hline Energy band $[\mathrm{GeV}]$ & Full mission $[\sigma]$ & MJD 58605.6-58855.6 $[\sigma]$ \\
\hline $0.1-0.316$ & 0 & 0 \\
$0.316-1$ & 1.50 & 0 \\
$1-3.16$ & 2.82 & 2.15 \\
$3.16-10$ & 4.20 & 1.07 \\
$10-31.6$ & 3.35 & 2.76 \\
$31.6-100$ & 0. & 0 \\
$100-316$ & 2.62 & 0 \\
\hline
\end{tabular}

and $\mathcal{T} \mathcal{S}_{2920 \text { d }}$ are the live time and significance of the source in the 4FGL catalogue, respectively. We note that in general for the significance $\Sigma=\sqrt{\mathcal{T S}}$. The source is detected with a significance of $5.42 \sigma$ in the 4FGL catalogue and hence the resulting integration times for one and two sigma are 100 days and 400 days, respectively. In order to avoid washing out a possible time-dependent signal we chose an integration time of 250 days. Table A.3 gives the significance of all $\gamma$-ray data points shown in the SED in Fig. 2. 